\title{
Piecewise Constant Feedback Control of Piecewise Affine Gene Network Models
}

\author{
Etienne Farcot and Jean-Luc Gouzé \\ COMORE INRIA, UR Sophia Antipolis \\ 2004 route des Lucioles \\ BP 93, 06902 Sophia Antipolis, France \\ \{etienne.farcot, gouze\}@sophia.inria.fr
}

\begin{abstract}
The use of hybrid dynamical systems to model gene regulation is impelled by the switch-like behaviour of the latter. Piecewise affine differential equations is one of the most extensively studied among such kind of models. We propose an extension of this class, introducing some input variables. A special focus is given to degradation and production rates being affine functions of the inputs. Some generic control problems are proposed, formulated in terms of an underlying discrete structure. Piecewise constant feedback laws that solve these problems are characterized in terms of affine inequalities. These general feedback laws are then applied to a well-known two dimensional example: the toggle switch. It is shown how to control this system toward various behaviours, especially bistability and bisimilarity with a discrete quotient.
\end{abstract}

\section{Introduction}

This work deals with control theoretic aspect of a class of piecewise-affine differential equations, introduced in the 1970's 5] to model gene networks, and since widely studied, both theoretically [5], and to model concrete biological systems 29]. Furthermore, recent advances have shown that gene networks may be synthesized in labs 48. This fact strongly motivates the elaboration of a control theory for such systems 68 variables are added to the original models to represent some physico-chemical influence on production and degradation rates. Among concrete realizations, one may use specific inhibitors or activators, introduced in a chosen quantity. Other techniques, such as directed mutagenesis, the use of interfering RNA [7], would also fit within the present framework. Mathematically, similar approaches have been proposed for multiaffine dynamical systems on rectangles [1, which would apply here, but these more general techniques are much less efficient than the specific methods introduced here. More details and references can be found in [3].

\section{Piecewise Affine Models}

The general form of models considered here is:

$$
\frac{d x}{d t}=\kappa(x, u)-\Gamma(x, u) x .
$$

A. Bemporad, A. Bicchi, and G. Buttazzo (Eds.): HSCC 2007, LNCS 4416, pp. 688692, 2007.

(C) Springer-Verlag Berlin Heidelberg 2007 
The state vector $x$ represents (mRNA or protein) concentrations: $x_{i}$ is the expression level of the $i$ th gene among $n$. Then, $u \in \mathcal{U} \subset \mathbb{R}_{+}^{p}$ is an input variable, meaning that additional biochemical species can be introduced in the system, or that some physical parameter (e.g. light intensity or temperature) is modified. In any case, input variables are bounded: $\mathcal{U}=\prod_{j=1}^{p}\left[0, U_{j}\right]$.

$\kappa(x, u) \in \mathbb{R}_{+}^{n}$ is a production term, and $\Gamma(x, u)$ a diagonal matrix, with positive diagonal entries $\gamma_{i}$ representing degradation rates. For a fixed $u$, both are piecewise constant functions of $x$ with a rectangular underlying partition, due to the switch-like nature of gene regulation.

Succinctly, the continuous dynamics is as follows: in any rectangle $\mathcal{D}$ in phase space, all trajectories are explicitly known, and tend toward a so-called focal point $\phi(\mathcal{D}, u)$. If $\phi(\mathcal{D}, u) \in \mathcal{D}$, one has a stable steady state and the system stays in $\mathcal{D}$ forever. Otherwise, the system leaves $\mathcal{D}$ in finite time, reaching another rectangular region. Repeating this provides well defined trajectories. This naturally leads to a discrete quotient of the dynamics, often called state transition graph, denoted $\mathrm{TG}(u)$ : rectangular regions are the states of this discrete system, and its transitions are defined as those pairs $\left(\mathcal{D}, \mathcal{D}^{\prime}\right)$ such that at least one continuous trajectory crosses $\mathcal{D}$ and $\mathcal{D}^{\prime}$ successively.

\section{Control Problems}

We focus on piecewise constant feedback control laws: $u=u(x)$, and the restriction $\left.u\right|_{\mathcal{D}}$ is constant for each $\mathcal{D}$. This relies on the assumption that threshold crossings, or switchings, can be detected accurately. Then, typical control problems are as below, where $\mathcal{V}$ denotes the set of vertices of TG, i.e. of rectangular regions in state space:

Global Control Problem: Let $\mathrm{TG}^{\odot}$ be a transition graph. Find a feedback law $u: \mathcal{V} \rightarrow \mathcal{U}$ such that $\mathrm{TG}(u)=\mathrm{TG}^{\odot}$.

Locally, at a region $\mathcal{D}$, solving this problem is tantamount to finding an input $u(\mathcal{D})$ such that $\phi(\mathcal{D}, u(\mathcal{D})) \in \mathcal{D}^{\prime}$, where $\mathcal{D}^{\prime}$ is easily deduced from the arrows in $\mathrm{TG}(u)$ having $\mathcal{D}$ as initial vertex. Since the vector $u(\mathcal{D})$ can be chosen arbitrarily in $\mathcal{U}$, this yields a controllable focal set, which is the whole set in which focal points can be chosen: $\phi(\mathcal{D}, \mathcal{U})$. A transition $\left(\mathcal{D}, \mathcal{D}^{\prime}\right)$ is then said controllable if $\phi(\mathcal{D}, \mathcal{U}) \cap \mathcal{D}^{\prime} \neq \varnothing$. This non-emptiness condition is equivalent to a system of affine inequalities, in the case when production and degradation terms are affine functions of $u: \Gamma(\mathcal{D}, u)=\operatorname{diag}\left(\Gamma(\mathcal{D}) u+\gamma^{0}(\mathcal{D})\right)$, and $\kappa(\mathcal{D}, u)=\kappa(\mathcal{D}) u+\kappa^{0}(\mathcal{D})$, where $\kappa(\mathcal{D}) \in \mathbb{R}^{n \times p}, \kappa^{0}(\mathcal{D}) \in \mathbb{R}_{+}^{n \times 1}, \Gamma(\mathcal{D}) \in \mathbb{R}^{n \times p}$ and $\gamma^{0}(\mathcal{D}) \in \mathbb{R}_{+}^{n \times 1}$.

A general form of local problem is then:

$$
\exists u \in \mathcal{U}, \forall i \in\{1 \cdots n\}, \theta_{i}^{-}<\phi_{i}(\mathcal{D}, u)<\theta_{i}^{+}
$$

and its solution is known. Denoting $T^{ \pm}=\operatorname{diag}\left(\theta_{1}^{ \pm} \cdots \theta_{n}^{ \pm}\right) \in \mathbb{R}^{n \times n}$, one gets

Proposition 1. An input $u$ solves problem $(P)$ if and only if it satisfies:

$$
\left\{\begin{array}{l}
\left(\kappa(\mathcal{D})-T^{-} \Gamma(\mathcal{D})\right) u>T^{-} \gamma^{0}(\mathcal{D})-\kappa^{0}(\mathcal{D}) \\
\left(\kappa(\mathcal{D})-T^{+} \Gamma(\mathcal{D})\right) u<T^{+} \gamma^{0}(\mathcal{D})-\kappa^{0}(\mathcal{D})
\end{array}\right.
$$


Two particularly interesting forms of transitions are depicted in figure [1 if all transitions in $\mathrm{TG}(u)$ are of one of these two forms, this graph yields a deterministic transition system, in which every path represents a continuous trajectory of the original system.

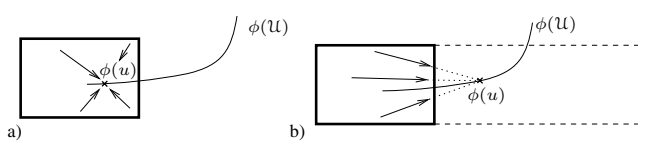

Fig. 1. Among all points in the focal set $\phi(\mathcal{U})$, one has to chose a particular $u$. Case a) is to make a region invariant, while in b), one has to find an input $u$ such that $\phi(u)$ is situated 'behind' a single facet of the box under consideration.

\section{The Toggle Switch Example}

One considers two genes inhibiting each other, often called toggle switch, and notably used as a building block of larger biological circuits 466]. Its biological function is that of a switch between two steady states, each being a long-term response to some transient induction. Here we suppose that the autonomous system is not bistable, and that a scalar input can affect the decay rates:

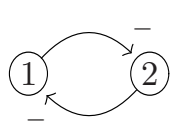

$$
\left\{\begin{array}{l}
\frac{d x_{1}}{d t}=\kappa_{1}^{1} \mathrm{~s}^{-}\left(x_{2}, \theta_{2}^{1}\right)+\kappa_{1}^{0}-\left(\gamma_{1}^{1} u+\gamma_{1}^{0}\right) x_{1} \\
\frac{d x_{2}}{d t}=\kappa_{2}^{1} \mathrm{~s}^{-}\left(x_{1}, \theta_{1}^{1}\right)+\kappa_{2}^{0}-\left(\gamma_{2}^{1} u+\gamma_{2}^{0}\right) x_{2}
\end{array},\right.
$$

where $\mathrm{s}^{-}(x, \theta)$ is the decreasing Heaviside (or step) function.

Solving a system of the form $(\star)$ at each of the 4 rectangular regions in this system, yields all the possible graphs TG $(u)$. Below are those obtained for a particular set of parameters, see 3] for details. The disposition of vertices reflects the geometry of state space, and $\mathbf{\square}$ represent regions containing a steady state.

a)

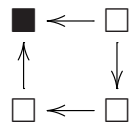

e)

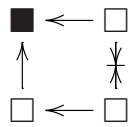

i)

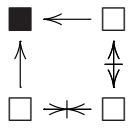

b)

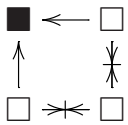

f)

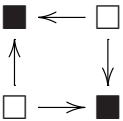

j)

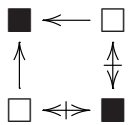

c)

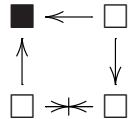

g)

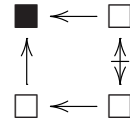

$k)$

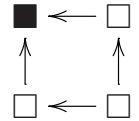

d)

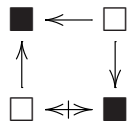

h)

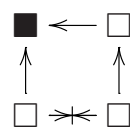

l)

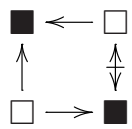

One can see that the upper-left vertex is always fixed, whatever the input value. The lower-right vertex, on the other hand, may be fixed or not. $a$ ) is the autonomous case. It appears that bi-stability may be ensured for transition graphs: 
$d), f), j$ ) and $l$ ). Another objective may be to require that the graph be deterministic, with transitions as in figure 1 only. Here, this may be achieved by inputs associated to the graphs $g$ ) and $j$ ). The first presents a single global equilibrium, while $j$ ) is bistable.

\section{Conclusion}

Among modern advances in cell biology, synthetic biology is one of the most striking and promising topic, which might involve control theoretic problems in the years to come. For gene regulatory networks modeled by piecewise affine differential equations, we have characterized the piecewise constant feedback laws that solve some qualitative control problems. An important follow-up of this work would be the analysis of more global control problems. In particular, instead of an explicit transition graph, one may aim at satisfying a formal property, like bi-stability or bisimilarity as exemplified in section 4 . Systematizing this with the aid of tools from model checking might lead to efficient algorithms. Also, the input $u$ may model uncertainties of the system. In particular, a property that holds for all $u$ (or whose negation is not controllable) could be called robust with respect to uncertainties, like for example the existence of at least one steady state in section 4.

Acknowledgments. This work was partially supported by the European Commission, project Hygeia Nest-004995. The authors would like to thank D. Ropers and H. de Jong for their helpful advice during the elaboration of this paper, and the referees for their suggestions.

\section{References}

1. C. Belta, L. Habets, V. Kumar, Control of multi-affine systems on rectangles with applications to hybrid biomolecular networks, 41st IEEE Conference on Decision and Control, pp. 534-539 (2002).

2. G. Batt, D. Ropers, H. de Jong, J. Geiselmann, R. Mateescu, M. Page, D. Schneider, Validation of qualitative models of genetic regulatory networks by model checking: Analysis of the nutritional stress response in Escherichia coli, Bioinformatics, 21(Suppl 1):i19-i28, (2005).

3. E. Farcot, J.-L. Gouzé, How to control a biological switch: a mathematical framework for the control of piecewise affine models of gene networks, Research Report RR5979, INRIA Sophia-Antipolis, https://hal.inria.fr/inria-00094853 (2006).

4. T.S. Gardner, C.R. Cantor, J.J. Collins, Construction of a genetic toggle switch in Escherichia Coli, Nature, 403:33 9-342 (2000).

5. L. Glass, Combinatorial and topological methods in nonlinear chemical kinetics, J. Chem. Phys. 63:1325-1335 (1975).

6. Hasty, J., Isaacs, F., Dolnik, M., McMillen, D., Collins, JJ, Designer gene networks: towards fundamental cellular control, Chaos 11, 207-220 (2001). 
7. F.J. Isaacs, D.J. Dwyer,J.J. Collins, RNA synthetic biology, Nature Biotechnology $24,545-554(2006)$

8. Kobayashi H, Kaern M, Araki M, Chung K, Gardner TS, Cantor CR, Collins JJ, Programmable cells: interfacing natural and engineered gene networks, Proc. Natl. Acad. Sci. U.S.A., 101(22):8414-9 (2004).

9. D. Ropers, H. de Jong, M. Page, D. Schneider, J. Geiselmann, Qualitative simulation of the carbon starvation response in Escherichia coli, BioSystems, 84(2):124-152 (2006). 